\title{
Characteristics of ArF Immersion Lithography Using Topcoat
}

\author{
Takeo Ishibashi ${ }^{1}$, Tetsuro Hanawa ${ }^{1}$, Toshifumi Suganaga ${ }^{1}$, Shinroku Maejima ${ }^{1}$, \\ Koichiro Narimatsu ${ }^{1}, K^{2}$ azuyiki Suko ${ }^{1}$, Mamoru Terai ${ }^{2}$, Teruhiko Kumada ${ }^{2}$, \\ Tomoyuki Ando ${ }^{3}$ \\ ${ }^{\prime}$ Renesas Technology Corp., 4-1 Mizuhara, Itami-shi, Hyogo 664-0005, Japan \\ ${ }^{2}$ Mitsubishi Electric Corp., 8-1-1 Honmachi, Tsukaguchi, Amagasaki-shi, Hyogo \\ 661-8661, Japan \\ ${ }^{3}$ Tokyo Ohka Kogyo Co., Ltd., 1590 Tabata, Samukawa-machi, Koza-gun, \\ Kanagawa 253-0114, Japan
}

\begin{abstract}
This paper is intended as investigations of two interesting characteristics of ArF immersion lithography using topcoat (TC). On the one hand, we identified several hundreds gel-type defects over large areas of the unexposed top surface of the resist in cases where a TC was used. On the other hand, we observed that the optical proximity effect (OPE) profiles in immersion lithography varied with the type of TC materials beyond expectation. The magnitude of OPE was reduced to approximately $20 \mathrm{~nm}$ in the region of maximum influence. We investigated the influences on these characteristics of the TC material and the methods of application (i.e. baking conditions and exposure). The chemical interaction (e.g., mixing) between the resist and the TC was examined by employing time-of-flight mass spectrometry with gradient shaving preparation (GSP + TOF) and FT-IR measurements and by focusing on the interface of the resist and TC. We confirmed consistent correlations between lithographic properties and chemical interaction. The TC material and process which form much mixing layer due to the chemical interaction tended to cause gel-type defect and variation of OPE profile.
\end{abstract}

Keyword: immersion lithography, topcoat, defectivity, optical proximity effect

\section{Introduction}

ArF immersion lithography is expected to become the most dominant technology for the production of devices with dimensions less than 65 nm. In recent times, this technology has progressed significantly. Last year, the fundamental optical performances of lithography were confirmed experimentally by using a trial production machine. Because its capability in experimental production has also been proved [1-2], it is crucial to take a decision on the need for TCs in mass production. However, the possibility of damage to the projector lens in the exposure tool by the components eluted from the resist material to the immersion water is still controversial. The establishment of a process technology that uses a TC to prevent elution is the most practical method to avoid such a damage in view of the enormous amount of investment involved [3-4]. In order to maximally exploit the TC material and process, it is necessary to develop a TC that has high compatibility with resists. It has been recognized that a high receding contact angle (CA) of water is required to maintain a stable meniscus essentially [5]. Moreover, the mechanism responsible for other phenomena arising from the usage of a TC must be examined. Although studies have been conducted on the mixing between several resist and TC combinations [6-7], very little attention has been paid to the influence of the mixing phenomena on lithographic performance. In this study, we would like to explore the influence on defectivity and OPE behavior of several types of TC materials and the corresponding methods of application. The chemical interaction at the interface of resist and TC needs to be understood. We shall discuss in detail the correlation between the process performance and the analytical results. 


\section{Experimental}

\subsection{Materials}

All experimental samples were supplied by Tokyo Ohka Kogyo Co., Ltd. in Kanagawa Japan. Three samples-Resist-A, Resist-B, and Resist-C-that comprise a methacrylate polymer were used in the OPE experiment. Only Resist-A was used in the study of defects. Two types of TC materials, hereafter referred to as TC-A and TC-B, were mainly examined in this experiment. The standard process condition for TC-A was set at 140 $\mathrm{nm}$ of film thickness and $60 \mathrm{sec}$ of soft baking (SB) at $90{ }^{\circ} \mathrm{C}$; this is a baking treatment applied after coating the resist with a TC. The condition for TC-B was set at $35 \mathrm{~nm}$ of film thickness and SB at $110{ }^{\circ} \mathrm{C}$ for $60 \mathrm{sec}$ of $\mathrm{SB}$. Some modulated samples based on the TC-A were tested in the OPE experiment. Solvent exchange and acid addition methods were applied as modulation methods. Those modulated samples were listed in Table 1.

Table 1 Sample list for studying the impact of material parameters on OPE.

\begin{tabular}{|c|c|c|c|}
\hline No. & Sample name & Modulation item & Formulation \\
\hline 1 & TC-A & $-\cdots$ & Polymer A (lower fluorine content) \\
\hline 2 & TC-A1 & Additive & Perfluoroalkylsulfonic acid $(0.5 \%)$ \\
\hline 3 & TC-A2 & Additive & Perfluoroalkylsulfonic acid $(10.0 \%)$ \\
\hline 4 & TC-A3 & Solvent-B & High boiling point (vs standard solvent) \\
\hline 5 & TC-A4 & Solvent-C & Mixed solvent (added water-shedding solvent) \\
\hline 6 & TC-B & Polymer & Polymer B (higher fluorine content) \\
\hline
\end{tabular}

\subsection{Chemical analyses of resist/TC interface}

\subsubsection{GSP+TOF analyses}

The film thickness was set at $170 \mathrm{~nm}$ for Resist-A and at $35 \mathrm{~nm}$ for both the TCs in this experiment. SB was applied at the standard temperature for each TC. All the samples were processed until postexposure bake (PEB) at $150{ }^{\circ} \mathrm{C}$, regardless of the existence of exposure.

Time-of-flight secondary-ion mass spectrometry (TOF-SIMS) measurement was performed by using TFS-2000 (Physical Electronics Corp.) after gradient shaving preparation (GSP). The mass spectra of positive and negative secondary ion fragments were obtained using ${ }^{69} \mathrm{Ga}^{+}$as the primary ion with a mass range from 0 to $1000 \mathrm{amu}$. The analysis of the depth profile of each constituent element becomes possible by plotting the remarkable mass fragment intensity, which is significant against the depth.

\subsubsection{FT-IR analyses after removal of TC}

Fourier transform infrared (FT-IR) spectra were obtained to investigate the residual TC component on the surface of Resist-A; this was after the removal of TC by treating it with a $2.38 \mathrm{wt} \%$ tetramethylammonium hydroxide (TMAH) developer. The resist thickness was fixed at 100 $\mathrm{nm}$ in order to obtain a higher ratio for the thickness of the mixed layer by total film thickness after the removal. Many types of sample wafers were prepared with different combinations of the two types of TCs, the existence or nonexistence of PEB, and the existence or nonexistence of shallow exposure. The amount of residue after the treatment was monitored by the specific absorption of the TC component at $1107 \mathrm{~cm}^{-1}$.

\subsection{Confirmation of lithography performances}

\subsubsection{OPE verification}

A scanner manufactured by Canon Inc. was used as a 193-nm exposure tool (FPA-6000AS4; $\mathrm{NA}=0.85$ ). Another scanner manufactured by Nikon Corporation was used as the immersion exposure tool (EET; maximum NA $=0.85$ ). The dry scanner and the immersion scanner were operated in line with an ACT-8 and an ACT-12 coater/developer, respectively; these developers were manufactured by Tokyo Electron Ltd. (TEL). The developer was 2.38 wt $\%$ TMAH. An attenuated phase-shifting mask with a transmittance of $6 \%$ was used. The illumination condition was $\sigma=0.85$ and NA $=0.85$. Three types of resist and nine types of TCs were used in this experiment. The detailed sample composition is described in the Result section. ARC-029A manufactured by Nissan Chemical Industries, Ltd., was used as a bottom antireflective coating. An OPE evaluation was performed using an isolated space pattern of 90 $\mathrm{nm}$ with various pitches. A secondary electron microscope (SEM) called S-9380 from Hitachi High-Technologies Corp. was used for the measurement of the resist pattern size.

\subsubsection{Verification of defectivity}

The standard process condition of TC-A and TC-B described above was used. A scanner manufactured by Nikon Corporation was used as the immersion exposure tool (EET; NA $=0.85$ ). The immersion exposure was operated by using an in-line construction with an ACT-12 (TEL). The developer was $2.38 \mathrm{wt} \%$ TMAH. An attenuated phase-shifting mask with a transmittance of $6 \%$ (i.e., 6\% HT) was used. The illumination condition was set at $\sigma=0.85$ and NA $=0.85$ for the $110-\mathrm{nm}$ line-and-space (L/S) pattern. This area of the defect test mask was $9.0 \mathrm{~mm} 2$; this comprised a $110-\mathrm{nm} \mathrm{L} / \mathrm{S}$ area $(7.6 \mathrm{~mm} \times 9.0 \mathrm{~mm})$, 
a $6 \%$-HT covered area $(0.5 \mathrm{~mm} \times 9.0 \mathrm{~mm})$, and a clear glass area $(0.9 \mathrm{~mm} \times 9.0 \mathrm{~mm})$. Two types of typical TC materials were used in the defect experiments. AR26 manufactured by Rohm and Haas Co. was used as a bottom antireflective coating. Defect inspection was carried out by the combination of array and random mode tests by using KLA2351. Defects detected in the both test modes were observed in order to classify into four categories by using SEM (S-9380).

\section{Results}

\subsection{Analytical results}

3.1.1. Results of GSP+TOF analyses

We investigated the chemical interaction at the boundary of the resist and TC by GSP + TOF analysis. Fig.1 shows the result of the film stack of Resist-A and TC-A without exposure. The horizontal axis shows the depth of the film stack. The dotted line at $0 \mathrm{~nm}$ marks the starting point of gradient shaving, i.e., the top surface of the TC. The dotted line at $35 \mathrm{~nm}$ shows the boundary between the TC and resist. This figure shows the concentration distribution of the components of the $\mathrm{TC}$ and resist around the interface. The significant components of the resist are the fragments derived from the polymer and the photoacid generator (PAG). The only significant fragment of the TC is the polymer. This analysis makes it possible to study the mutual movement of the components of each film: that is diffusion in the other word.

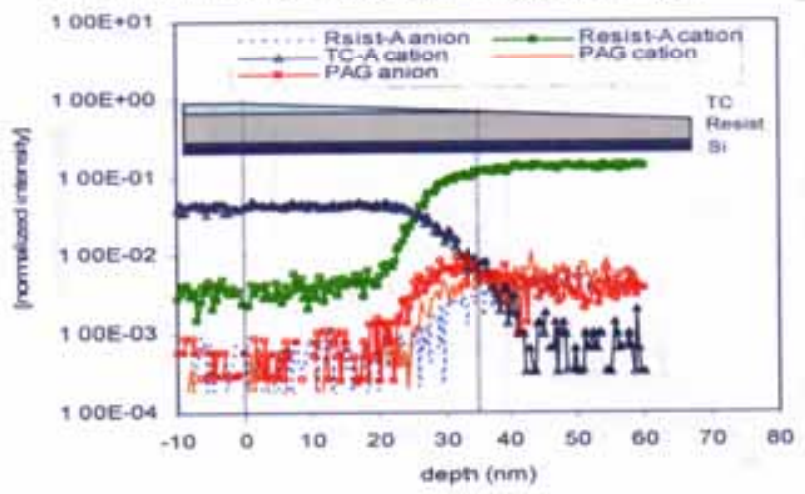

Fig.1 Result of the GSP + TOF analysis of the film stack composed of Resist-A and TC-A without exposure.

We examined the influence of mimic immersion exposure on the film stack composed of Resist-A and TC-A. The dose was set at the optimum energy for a $110-\mathrm{nm} \mathrm{L} / \mathrm{S}$ pattern. As depicted in Fig.2, the fragments of the protection group and the resist polymer show a gradual slope into the TC layer. This proves the formation of a mixing layer between the TC and the resist. Although no change in the fragments of the resist polymer was observed irrespective of the application of exposure, the intensity of the fragment of the protection group of the exposed sample becomes lower than that of the unexposed one. This is a very reasonable behavior related to the deprotection reaction that occurs due to the exposure.

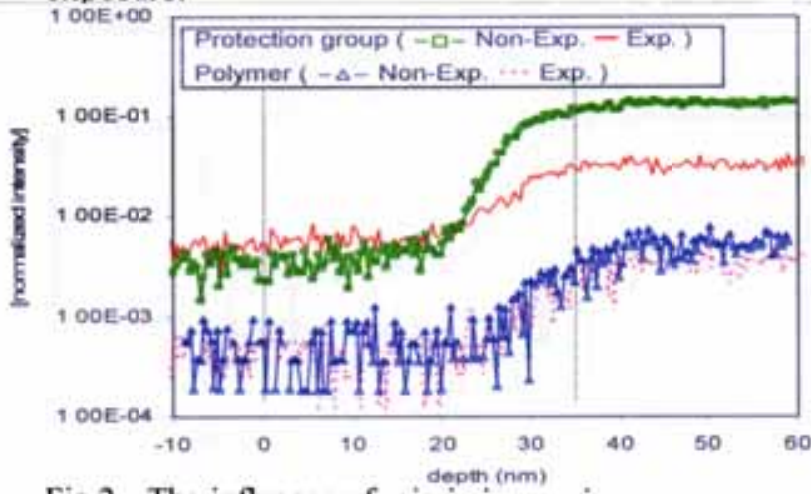

Fig.2 The influence of mimic immersion exposure on the distribution of the polymer fragments of Resist-A in $\mathrm{TC} /$ resist film stack.

Fig. 3 shows the behaviors of PAG components with regard to exposure or nonexposure. This result indicates two reasonable facts. First, the fragment of the PAG cation disappeared after exposure because of photodecomposition reaction. Second, the fragment of the PAG anion derived from the exposed sample penetrated [Note: Please check the change.] the TC film significantly compared with that resulting from the unexposed sample. This phenomenon is termed "elution to TC."

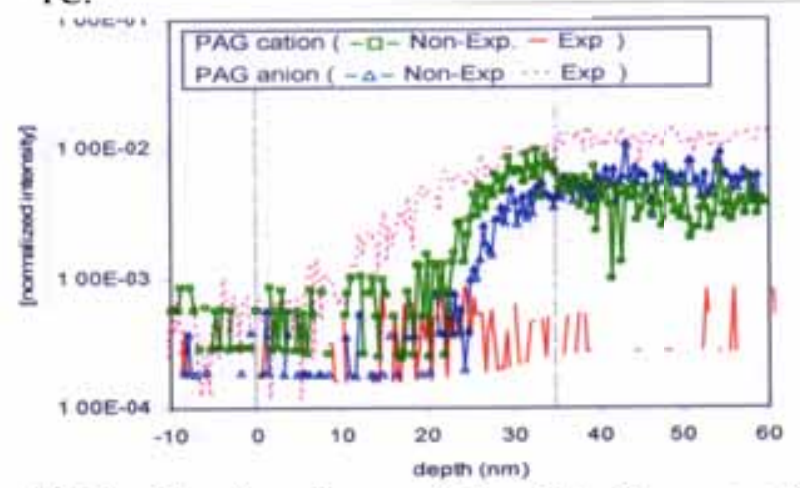

Fig. 3 The dependency of the PAG fragment of Resist-A on mimic immersion exposure in the GSP + TOF analysis.

Moreover, we focused attention on the difference between the two types of TC materials as an interesting subject. As shown in Fig.4, both the fragments derived from the resist polymer and PAG diffused slightly into the resist film when TC-B was coated on Resist-A. 


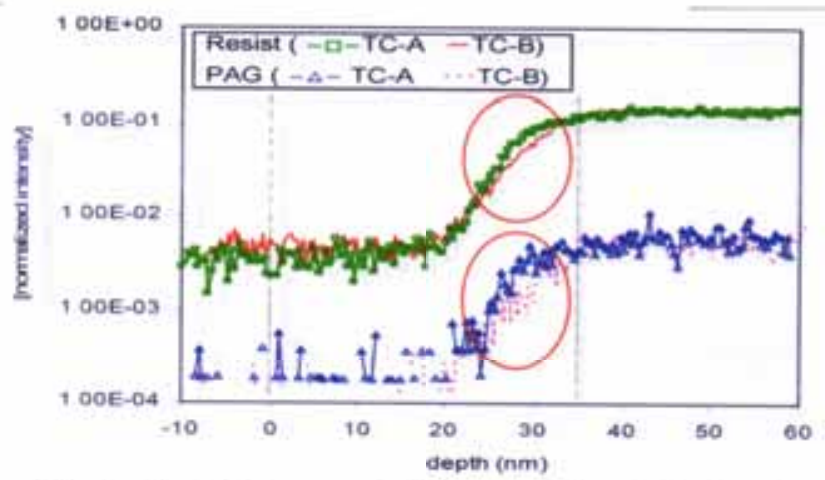

Fig.4 The influence of different kind of TC for the distribution of the polymer fragment of Resist-A in TC/resist film stack with only mimic water immersion without exposure.

We analyzed PAG elution into the TC by using two types of resists without exposure, as shown in Fig.5. The PAG of Resist-A was the salt of triphenylsulfonium (TPS)-like cation and nonaflate anion and that of Resist-C was a TPS-like cation and triflate anion. As a result, the smaller molecule of triflate-TPS (i.e., Resist-C) exhibited a greater elution into TC despite the absence of exposure. This elution will have some effect on mixing between the resist and TC, as discussed below.
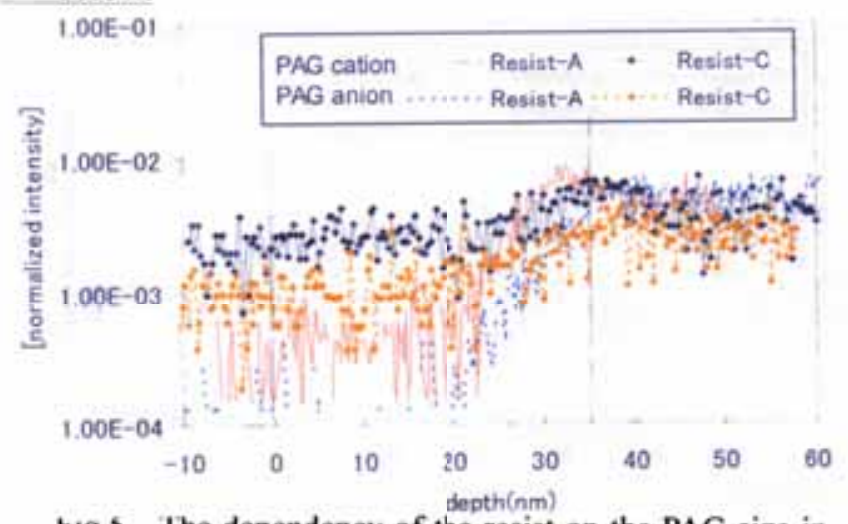

rig.5 The dependency of the resist on the PAG size in the GSP+TOF analysis.

\subsubsection{Results of FT-IR analyses}

The FT-IR measurements were performed to estimate the amount of residues of the TC component on the resist surface after removal by TMAH development. The samples prepared for this experiment are listed in Table 2.

For example, the amount of residue resulting from the SB + PEB treatment of the film stack composed of TC-A/Resist-A was estimated by calculating the difference in absorbance at 1107 $\mathrm{cm}^{-1}$ between sample 4 and sample 1 (as a reference) in Table 2. The IR chart for samples 1, 2 , and 4 are shown in Fig.6. The slight broad absorption at $1107 \mathrm{~cm}^{-1}$ was attributed to the peak resulting from the $\mathrm{C}-\mathrm{F}$ bond, which is common to in the IR chart of both the TCs.

Table 2 The sample list for the FT-IR measurement
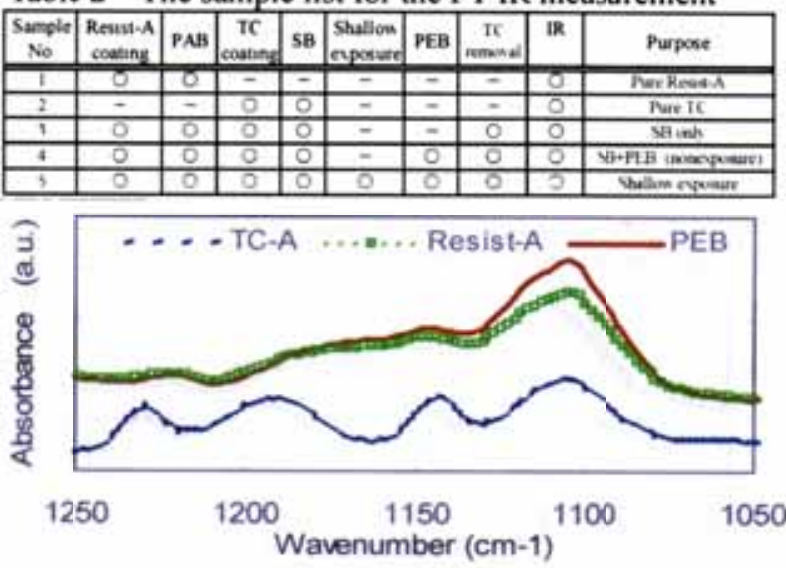

Fig.6 Example of a FT-IR chart used to estimate the amount of residues.

Relative increases in the amount of residues, which were normalized by the absorption of pure Resist-A at $1107 \mathrm{~cm}^{-1}$, are summarized in Fig.7. Fig.7 (a) indicates that the mixing layer appears to be formed at the time of the SB process. In other words, PEB is not as effective as SB for the formation of the mixing layer. It was observed from the result that TC-A forms more mixing layers compared with TC-B in both the cases of nonexposure (Sample 4) and shallow exposure (Sample 5), as shown in Fig.7 (b). (a)

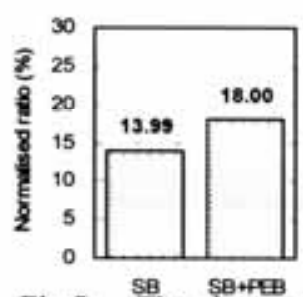

(b)

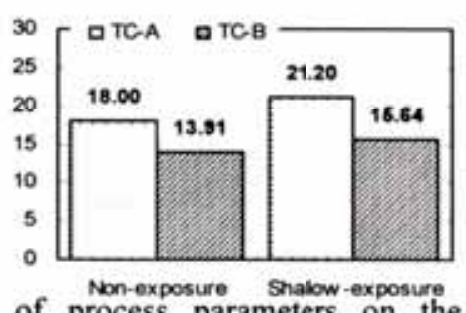

Fig.7 The impact of process parameters on the amount of residue after TC removal, as analyzed by FT-IR.

(a) Comparison between baking processes using TC-A

(b) Comparison between the amount of residue of the TCs.

\subsection{Results of lithography performances}

\subsubsection{Results of defect inspection}

The wafer maps of the defect inspection results with respect to three combinations of TCs and resists before classification of the defect categories are shown in Fig.8. Array mode inspection was applied to the L/S pattern region; random mode inspection was applied to the HT-covered region and the clear glass region. These results indicate that the defect count is influenced by both the TC and resist materials. The overviews of the wafer 
maps have no special characteristics such as misdistribution of defects.

(a)
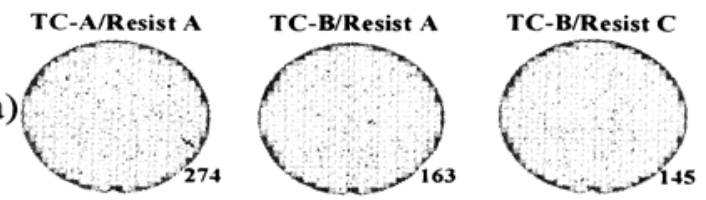

(b)
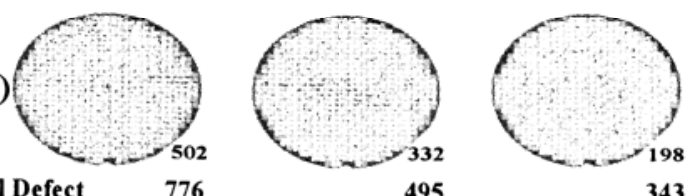

495

343

Fig. 8 Wafer defect maps; (a) L/S pattern region tested by array mode, (b) HT covered and clear glass regions tested by random mode.

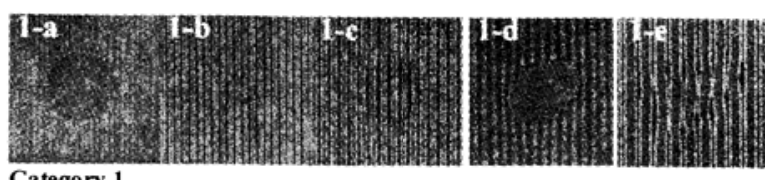

Category 1

Circular and deformed circular shape defects.

1-a: Circular shape large bridge. 1-d Non circular shape large bridge

1-b Circular shape line pitch narrowing 1-e: Non circular shape line deformation

1-c. C. ircular shape line line pitch enlarging
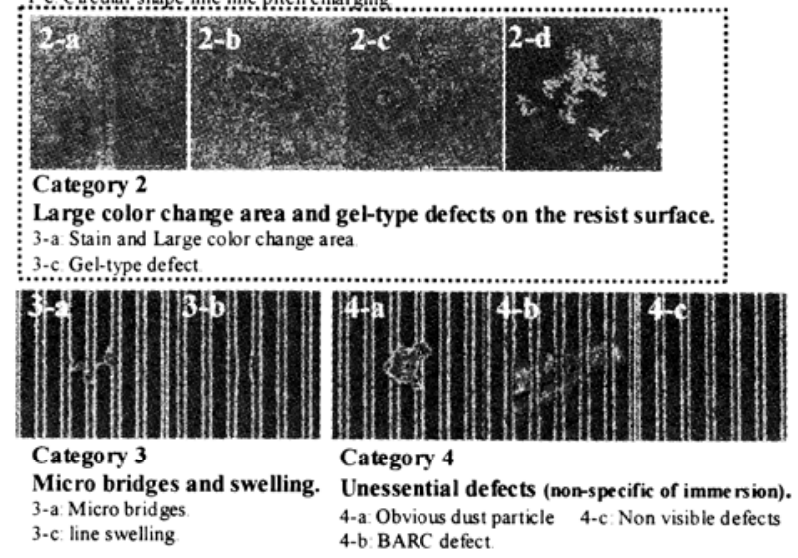

Fig.9 The pictures of the defect of each categories. The most significant defects classified into Category 2 in this study were observed on the surface of the resist.

Because the counts of defects in Fig.8 were values without utilizing auto-clustering function of KLA2351, there is a possibility of the multi (i.e. over) countings of a large defect. All the detected defects (in both types of inspection regions) were classified into four types of defect modes, as summarized in Figs.10 and 11. The classification is as follows.

The Category 1 is derived from water droplets generally; the Category 2 is derived from the chemical interaction between the resist and TC resulting in a gel-type defect; the Category 3 comprises micro-bridge and line-swelling which is non-specific defects of immersion lithography, that is observed in conventional dry lithography for a non-immersion case; and the Category 4 comprises

unessential defects that is non-visible defect, obvious dust particle and defect originated in BARC film.

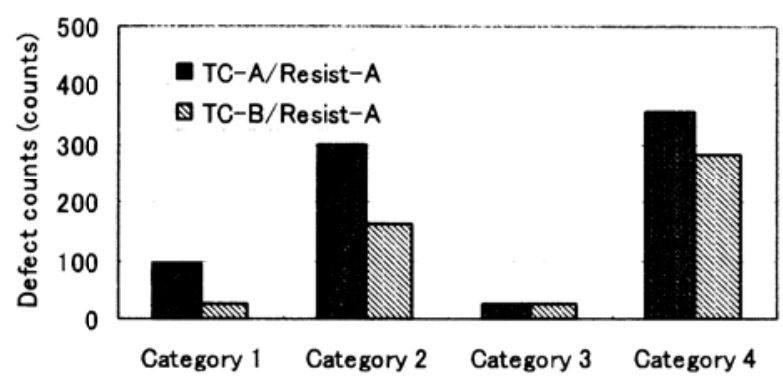

Fig.10 The classified defect counts of TC-A and TC-B on Resist-A

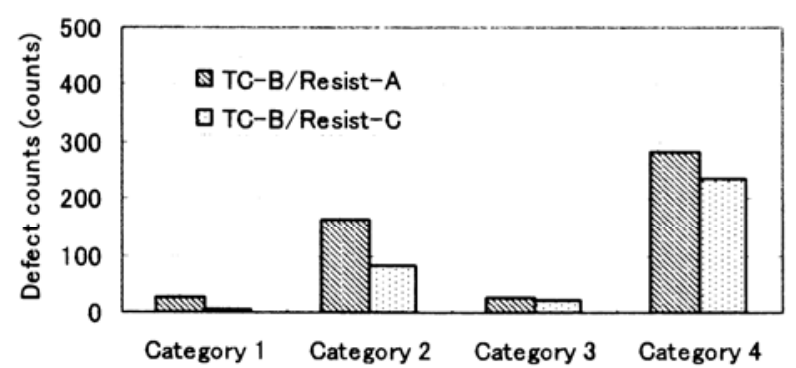

Fig.11 The classified defect counts of Resist-A and Resist-C under TC-

The significant defects were those of Categories 1 and 2. The static and receding CAs of TC-A and TC-B were measured. Because TC-B is composed of a polymer of higher fluorine content, its CA is greater than that of TC-A. In particular, the values of the receding CA for TC-B and TC-A were $68.1^{\circ}$ and $58.9^{\circ}$, respectively. Based on the comparison of receding CAs, the defect classified in Category 1 could be improved because a higher receding CA prevents the formation of water droplets [5].

Category 2 defects showed an apparent dependency not only on the type of TC but also on the type of resist material. This is natural because Category 2 defects are derived from the interfacial mixing between the TC and resist. This result of the mixing (i.e., chemical interaction) will be discussed in detail in the Discussion section.

\subsubsection{Results of OPE verification}

We verified the behavior of OPE in ArF immersion lithography by initially coating TC-A and TC-B on Resist-B. The profiles of the OPE for the two process conditions are shown in Fig. 12. The result clearly indicated that the profile of OPE depended on the TC material and process. 


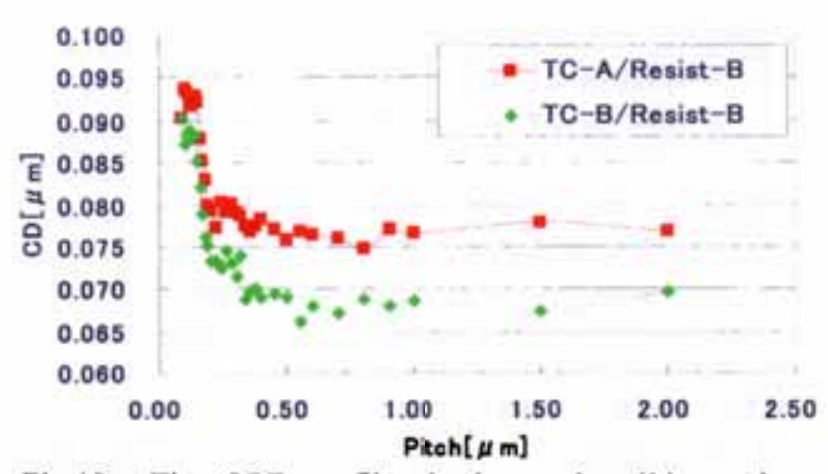

Fig.12 The OPE profiles in immersion lithography using TC.

We performed many experiments to investigate the dependency of OPE on process and material parameters by using the dry lithography process. We suspected that the OPE would be affected by the difference in the standard process parameters of each TC. Therefore, we examined the dependence of OPE on the TC process conditions, as shown in Fig.13. The thickness and SB temperature of a TC might change the profile of OPE due to optical interference and chemical effects. However, the result indicated that these process conditions were not the major factors responsible for OPE variation when either TC was used.

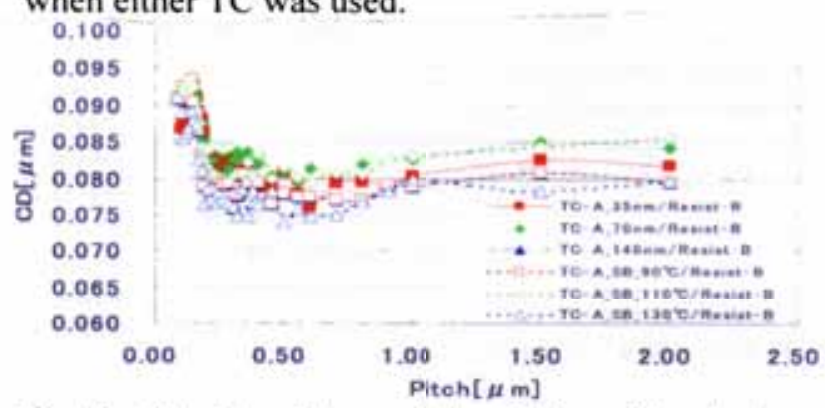

Fig.13 The dependency of the OPE profiles in dry lithography using TC on the process condition.

Next, let us examine the OPE variation with regard to the type of material parameters. We prepared three types of resist and five types of TCs for this experiment as listed in Table 1. The OPE profiles of a variety of TCs are shown in Fig.14. In particular, in the case of Resist-A, the formulation of the TC material had a considerable impact on OPE.

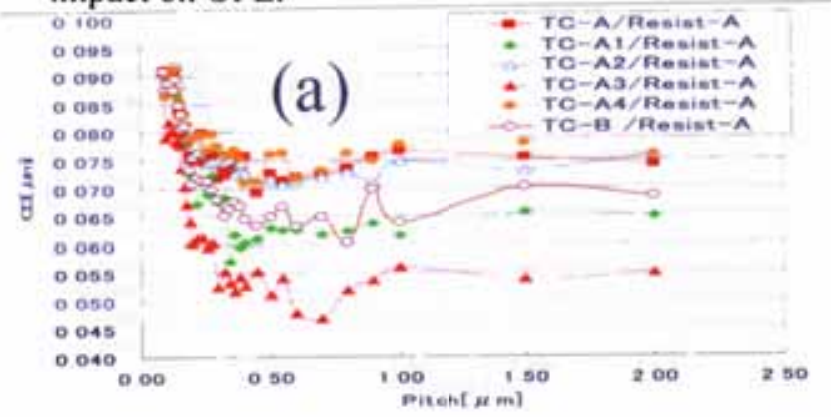

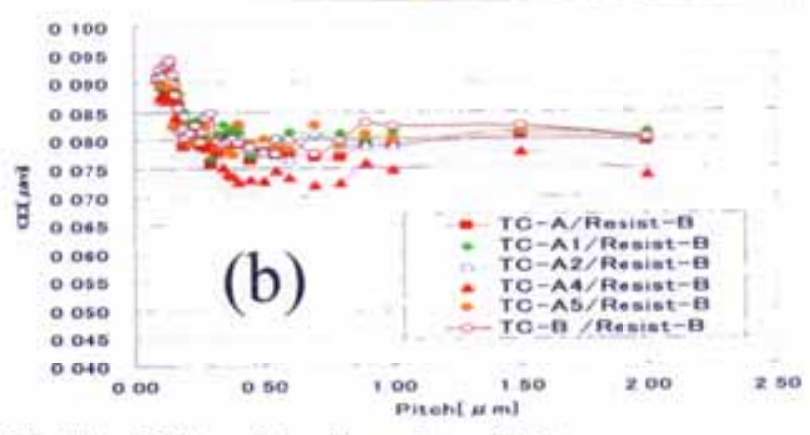

Fig.14 OPE profiles of a variety of TCs.

(a) With Resist-A (b) With Resist-B.

One of the effective material parameters was acid addition with enough amount (i.e. $10 \%$ ). Addition of perfluoroalkyl sulfonic acid, which is not suitable for practical method because of the problem of elution from TC in itself, was thought to affect the chemical reaction at the time of baking. The OPE profile of Sample 3 (TC-A2) was almost similar to that of TC-B.

Another significant parameter that showed a considerable degree of impact was solvent exchange. The TC solvent should control the mutual affinity between the resist and TC in order to control the diffusion between them. We studied the interaction between the resist and the solvents by measuring the change in the physical properties of the resist surface. Thickness losses were not found for any solvent. Hence, we measured the CA of water on the resist surface after dipping the resist in the solvents described in Table 3.

Table.3 The CA change with the solvent treatment

\begin{tabular}{|c|c|c|c|c|}
\hline & Ponionth & $\begin{array}{l}\text { Doped in } \\
\text { tonderd whemt }\end{array}$ & $\begin{array}{l}\text { Oxpes by } \\
\text { solvente }\end{array}$ & $\begin{array}{l}\text { Doped n } \\
\text { soterertic }\end{array}$ \\
\hline Corbest mole & 654 & 608 & 613 & 680 \\
\hline
\end{tabular}

It was observed that solvent treatment imparted a hydrophilic or hydrophobic property to the surface. Although this investigation was not sufficient to draw a definite conclusion, we found that the solvent changes the affinity of the surface with regard to the developer solution; this consequently changes OPE profiles.

The material parameters of TC did not have a big impact on Resist-B despite a considerable impact on Resist-A. This feature appears to be concerned with the difference in the acid movement, as shown in Fig.5.

The cross-sectional view of an isolated pattern, which shows considerable differences in the OPE profile, was observed by SEM. In this figure, we can recognize that the difference in the OPE profiles originated from the interaction between the TC and resist. 

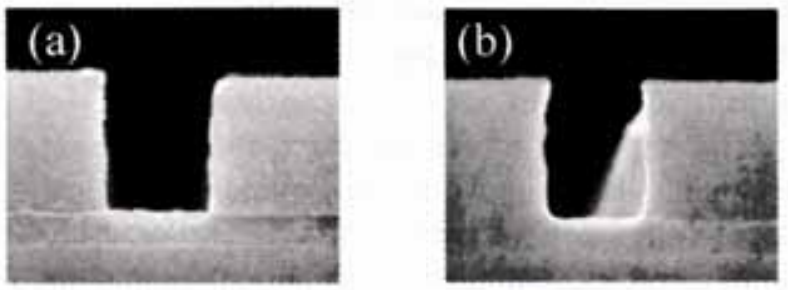

Fig.15 Cross sections of samples with large and small OPE range. (a) TC-A (b) TC-A3 on Resist-A

According to another perspective, the degree of OPE variation could be controlled by appropriately selecting the TC material. As Fig.16 indicates, the OPE profiles obtained using TC-B were independent of the three resist materials that we used. The bold line is indicative of the result of the simulation of an optical image in the resist with the consideration of the blur due to resist chemistry (Prolith, Ver. 9.2).

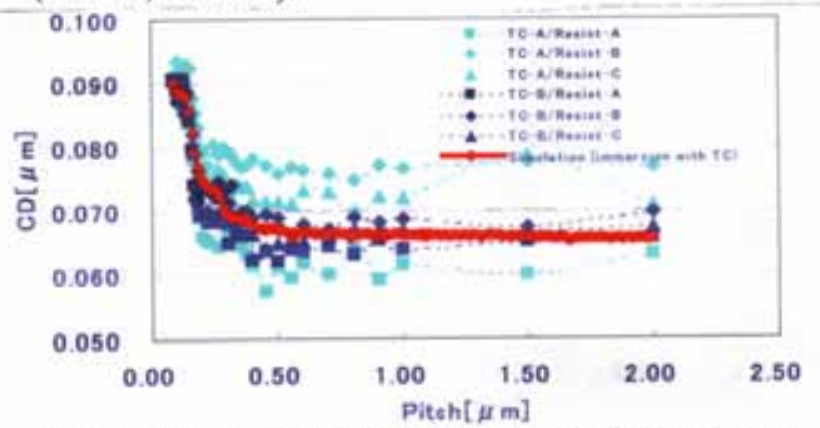

Fig.16 The dependency of the OPE profiles in dry lithography on the type of TC material.

Vibration was set at $20 \mathrm{~nm}$ as the standard deviation to take into account the blur caused by acid diffusion mainly. This value of the standard deviation corresponds to $4.5 \mathrm{~nm}$ of the average diffusion length expressed by $\sqrt{2 D t}$ if the blur was composed only of acid diffusion. The OPE profile obtained by simulation was very similar to that obtained using TC-B. This means that the OPE profiles of TC-B should be determined without any chemical effects.

\section{Discussion}

We would like to discuss the influence of chemical interaction, which is simply referred to as "mixing," on defect formation and OPE profiles. Chemical interaction includes not only the mutual diffusion of the components of each film but also the chemical reactions of the diffused components.

\subsection{Correlation between defects and mixing}

In our study, it was observed from the analysis of GSP + TOF that there was a movement of chemical components through the film interface. Moreover, we confirmed that the movement depended on the type of TC: TC-A showed a higher tendency toward chemical interaction than TC-B. A similar result indicating that TC-A forms more mixing layers was obtained in the analysis of residues by FT-IR.

On the other hand, a few hundred gel-type defects were observed in the unexposed HT-covered region in the evaluation of defectivity. The defect count of TC-A was two times larger than that of TC-B. This result was consistent with those obtained in the chemical analyses mentioned above. The gel-type defect can be tentatively explained as follows. If the mixing layer has a very low solubility in TMAH, the polymer material dissolved in TMAH after considerable effort forms a suspended matter called gel. It reattaches easily to the resist surface, which has higher affinity to a gel; this reattachment consequently causes gel-type and large stain-type (i.e. large color change area) defects.

In the comparison between Resist-A and Resist-C, the latter appears to have an advantage with regard to defectivity. We are now assuming that the acid eluted from the resist to TC, which was proved by the GSP + TOF analysis shown in Fig.5, inhibited some chemical reactions in the baking process. The discussion on the OPE behavior described below is also based to some extent on this assumption.

\subsection{Correlation between OPE and mixing}

A dependency on the type of TC was observed in the investigation of the behavior of the OPE profiles. In the comparison of the OPE profiles of TC-A and TC-B, the latter showed a very consistent result that was independent of the resist type and almost identical to the result of the physical simulation. In contrast, the OPE profiles of TC-A differed considerably with the resists and showed no agreement with the simulation result. This also indicates a lower mixing in the case of TC-B than TC-A.

Let us now discuss the variation in the OPE profiles in detail. The results of solvent exchange, which was intended for changing the ability of mutual diffusion by tuning the affinity for each other, showed a considerable impact. This proved that variations in OPE were affected by chemical interactions. As focusing to the sample added enough acid (Sample 3), the OPE profile become similar to TC-B. We believe that this suggests the influence of acid (added on purpose) 
at the interface region between the resist and TC. We believe that the presence of the strong sulfonic acid will definitely have some influence on chemical reactions if there are chemical interactions at the interface. On the other hand, the addition of acid was not found to have a significant impact on the experiment with Resist-B. Because the composition of PAG is similar in Resist-B and Resist-C, an increased amount of PAG penetrates into the TC film in the absence of exposure, as shown in Fig.5. The rich eluted PAG is responsible for the stable OPE profile of the different TC materials. In other words, the presence of acid will impact mixing. The result obtained in the GSP + TOF analysis of the sample with sufficient exposure to form an 110-nm L/S pattern showed an apparent acid movement from the resist to the TC (Fig.3). Based on the result of acid movement, the degree of mixing is expected to depend on the amount of photo-generated acid (i.e., dose energy). Consequently, the OPE variations among the combinations of resists and TCs should be caused by a few factors as described below. The first is the affinity between the resist and TC which affects to the mutual diffusion and the wettability with respect to developer solution. The second is the amount of acid at the boundary of the resist and TC originated by decomposition of the PAG. The hypothesis that the mixing around the interface affects the OPE profile was supported by the cross-sectional profiles at the interface region of the samples with different OPE profiles, as shown in Fig. 15.

\section{Conclusion}

We investigated two interesting characteristics of ArF immersion lithography using TC, namely, defectivity and OPE. Both these characteristics were affected by the chemical interaction between the resist and TC. This interaction caused a solubility inhibition effect at the interface of the TC and resist. This inhibition results in the formation of a gel in the developer solution. It also results in a gel-type defect in the HT-covered region. The degree of chemical interaction appeared to be dependent on the presence of acid in the mixing region. This was concluded based on the experiment in which perfluoroalkyl sulfonic acid was added to the interface between the TC and resist. In the other cases, the samples with resist formulations, which can provide a considerable amount of acid from PAG to the TC, showed an improvement in defect and OPE. Furthermore, as TC-B showed less mixing, the characteristics of defectivity and OPE were very consistent.

It is important to prevent the mutual diffusion inherently by maintaining an optimum chemical affinity between the resist and TC in order to achieve a stable performance in immersion lithography using TC. We believe that a higher fluorine content and the selection of an optimum solvent in order to overcome the mixing problem should be the key to preventing mutual diffusion.

Finally, it must be noted that this inhibition effect is not a universal principle for all TC materials; thus, there is a possibility that it may promote dissolution. Therefore, there is a possibility to control the OPE profile flat and smooth without increasing gel-type defects due to the low solubility of the mixing layer.

\section{Acknowledgements}

The authors would like to thank Nikon Corporation for their technical support. They are also grateful to Tokyo Electron AT Ltd. for their cooperation in the immersion experiments.

\section{References}

1. D. Gil, T. Bailey, D. Corliss, M.J. Brodsky, P. Lawson, M. Rutten, Z. Chen, and N. Lustig, and T. Nigussie, Proc. of SPIE, 5754 (2005) 119.

2. Jeng-Horng Chen, Li.-Jui Chen, Tun-Y.ing Fang, et al., Proc. of SPIE, 5754 (2005) 13.

3. R. R. Dammel, G.. Pawlowski, A. Romano, Frank M. Houlihan, Woo-Kyu Kim, R. Sakamuri, D. Abdallah, M. Padmanaban, M.D. Rahman and D. McKenzie, J. Photopolym. Sci. Technol., 18 (2005) 593.

4. H. Tsuji, M. Yoshida, K. Ishizuka, T. Hirano, K. Endo and K. Ohmori, J. Photopolym. Sci. Technol., 18 (2005) 641.

5. T. Ando, K. Ohmori, S. Maemori, T. Takayama, K. Ishizuka, M. Yoshida, T. Hirano, J. Yokoya, K. Nakano, T. Fujiwara and S. Owa, "Defect Studies of Resist Process for 193nm Immersion Lithography", Proc. of SPIE (2006), to be published.

6. S. Kishimura, R. Gronheid, M. Ercken, M. Maenhoudt, T. Matsuo, M. Endo, and M. Sasago, Proc. of SPIE, 5753 (2005) 20.

7. S. Kanna, H. Inabe, K. Yamamoto, S. Tarutani, H. Kanda, K. Mizutani, K. Kitada, S. Uno and Y. Kawabe, J. Photopolym. Sci. Technol., 18 (2005) 60 